\title{
Development of highly sensitive mechanoluminescent sensor aiming at small strain measurement ${ }^{*}$
}

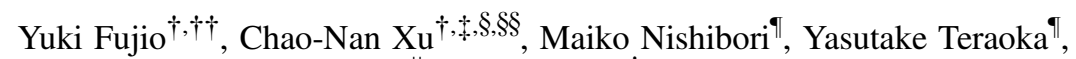 \\ Kazutaka Kamitanill, Nao Terasaki ${ }^{\dagger}$ and Naohiro Ueno** \\ ${ }^{\dagger}$ Measurement Solution Research Center \\ National Institute of Advanced Industrial Science and Technology \\ Saga 841-0052, Japan \\ *International Institute for Carbon-Neutral Energy Research (WPI-I ${ }^{2}$ CNER) \\ Kyushu University, Fukuoka 819-0395, Japan \\ $\S$ Interdisciplinary Graduate School of Engineering Sciences \\ Kyushu University, Fukuoka 816-8580, Japan \\ "Faculty of Engineering Sciences \\ Kyushu University, Fukuoka 816-8580, Japan \\ $\|_{\text {Research Center for Synchrotron Light Applications }}$ \\ Kyushu University, Fukuoka 816-8580, Japan \\ **Graduate School of Science and Engineering \\ Saga University, Saga 840-8502, Japan \\ ††yuki-fujio@aist.go.jp \\ $\S \S_{\mathrm{cn}-\mathrm{xu} @ \text { aist.go.jp }}$
}

Received 14 May 2014; Accepted 28 May 2014; Published 24 June 2014

\begin{abstract}
This paper was focused on the elasticoluminescence (ELS) characteristics, especially a response to small strain (below $1000 \mu$ st), of mechanoluminescence (ML) sensor using strontium aluminate doped with small amount of europium $\left(\mathrm{SrAl}_{2} \mathrm{O}_{4}: \mathrm{Eu}\right)$ synthesized by different methods. By using nitrate decomposition method as a synthetic method of $\mathrm{SrAl}_{2} \mathrm{O}_{4}$ : Eu, the response to small strain of the ML sensor was enhanced in comparison with using a conventional solid-state reaction method. Based on SEM observation and thermoluminescence $(\mathrm{ThL})$ measurement, we proposed a hypothesis that the sensing characteristic of small strain affect the platelike shape of $\mathrm{SrAl}_{2} \mathrm{O}_{4}$ :Eu grain and/or shallower carrier trap levels formed by nitrate decomposition method.
\end{abstract}

Keywords: Mechanoluminescence; $\mathrm{SrAl}_{2} \mathrm{O}_{4}:$ Eu; small strain measurement.

\section{Introduction}

A mechanoluminescence (ML) sensor using composite film prepared by using elasticoluminescence (ELS) material and flexible polymeric material is one of the promising candidates for health monitoring of various constructions, such as bridge, building, chemical plant, and social infrastructure, as well as an effective tool to check a risk and residual life of products. ${ }^{1-11}$ The sensing characteristics of ML sensor are mainly affected by the optical and structural properties of ELS material, e.g., emission intensity, emission wavelength, and anisotropic deformation. ${ }^{3,12-14}$ Among various ELS materials reported, strontium aluminate doped with small amount of europium $\left(\mathrm{SrAl}_{2} \mathrm{O}_{4}: \mathrm{Eu}, \mathrm{SAOE}\right)$ has been reported to give much stronger green light emission (about $520 \mathrm{~nm}$ ), which can be easily seen by our naked eye..$^{2,12,15}$
So far, there have been numerous reports regarding the improvement in ELS characteristics of a composite material consisting of SAOE powder and polymeric material. For example, Xu et al. have achieved that the ELS intensity was dramatically enhanced by controlling the amount of Sr vacancy and crystal structure. ${ }^{3,12}$ Subsequently Sohn et al. have reported to improve the ELS intensity by tuning the amount of dopant Eu ion. ${ }^{16}$ However, these reports were focused on the ELS intensity toward much larger stress (or strain), and as far as we know, there are few reports of a response to relatively small stress (or strain). This is a highly preferable characteristic in view of the actual application of the sensor to structural health monitoring and check tool of product. Such a highly sensitive response to a small mechanical stimulation, such as compression, tension, friction, and vibration, is strongly required to commercialize an ML sensor.

*Selected paper from The 2nd International Conference on Mechanoluminescence and Novel Structural Health Diagnosis, Tongji University, Shanghai, China, 10-13 November 2013.

This is an Open Access article published by World Scientific Publishing Company. It is distributed under the terms of the Creative Commons Attribution 3.0 (CC-BY) License. Further distribution of this work is permitted, provided the original work is properly cited. 
In the present study, the ML sensor utilizing SAOE films synthesized by different methods were fabricated and their ELS characteristics were examined, aiming at the development of highly sensitive detection of a small strain (below $1000 \mu \mathrm{st}$ ). After optimizing a synthetic method of SAOE powder, the influential factors for small strain response of the fabricated ML sensor were also investigated and discussed here.

\section{Experimental Procedure}

\subsection{Preparation of $\mathrm{SrAl}_{2} \mathrm{O}_{4}: \mathrm{Eu}$}

The sensing materials of SAOE were synthesized by a conventional solid-state reaction method and a nitrate decomposition method. The solid-state reaction method has been commonly used as a method for manufacturing $\mathrm{SrAl}_{2} \mathrm{O}_{4}: \mathrm{Eu}$. In this study, the commercial $\mathrm{SrCO}_{3}$ (Kanto Chemical Co., Inc., Japan), $\alpha-\mathrm{Al}_{2} \mathrm{O}_{3}$, and $\mathrm{Eu}_{2} \mathrm{O}_{3}$ (Kojundo Chemical Laboratory Co., Ltd., Japan) were weighed precisely to the molar ratios of $\mathrm{Sr}_{0.97} \mathrm{Al}_{2} \mathrm{O}_{4}: \mathrm{Eu}_{0.03}$, and mixed together by means of ball-milling method (using a planetary ball-mill, Pulverisette 7, Fritsch, Germany) in ethanol solution $\left(10 \mathrm{~cm}^{3}\right)$ at $600 \mathrm{rpm}$ for $2 \mathrm{~h}$ using zirconia balls $(3 \mathrm{~mm}$ in diameter). The resulting suspension was stirred and dried simultaneously to obtain a precursor of SAOE. In the nitrate decomposition method, the corresponding metal nitrates $\left(\mathrm{Sr}\left(\mathrm{NO}_{3}\right)_{2}\right.$ and $\mathrm{Eu}\left(\mathrm{NO}_{3}\right)_{3} \cdot 6 \mathrm{H}_{2} \mathrm{O}$ : Kanto Chemical Co., Inc., Japan; $\mathrm{Al}\left(\mathrm{NO}_{3}\right)_{3} \cdot 9 \mathrm{H}_{2} \mathrm{O}$ : Kojundo Chemical Laboratory Co., Ltd., Japan) were dissolved in distilled water. The aqueous solution was mixed well by ultra-sonication for $1 \mathrm{~h}$, and then vigorously stirred on a hot-plate stirrer and evaporated to dryness in an atmospheric air. The precursors obtained from different methods were calcined at $800^{\circ} \mathrm{C}$ for $1 \mathrm{~h}$ in air and sintered subsequently at $1350^{\circ} \mathrm{C}$ for $4 \mathrm{~h}$ in $5 \%$ $\mathrm{H}_{2} / \mathrm{Ar}$ atmosphere. The SAOE powders synthesized by solidstate reaction method and nitrate decomposition method are denoted by SS-SAOE and ND-SAOE, respectively.

Each of the synthesized SAOE powders was thoroughly mixed with epoxy resin to prepare SAOE pastes. The SAOE films (ML sensor) were fabricated by means of a screen printing technique using each resulting SAOE paste. The thickness of SAOE films was controlled to be approximately $30 \mu \mathrm{m}$.

\subsection{Evaluation of $M L$ sensor}

The ELS characteristics of the fabricated SAOE films were evaluated under a laboratory-built ELS evaluation system in a dark room at room temperature, as reported elsewhere. ${ }^{6,8,17}$ The ELS evaluation system is made up of metal test piece (SUS631, $200 \times 20 \times 0.5^{t} \mathrm{~mm}$ ) attached with an SAOE film $(10 \times 10 \mathrm{~mm})$ and a commercial strain gage (Kyowa Electronic Instruments Co., Ltd., Japan), a CCD camera, material testing machine (MTS 810, MTS Systems Co., US),
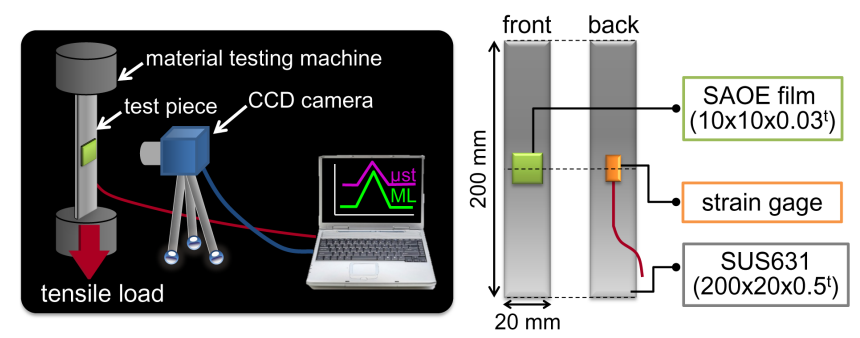

Fig. 1. The schematic illustration of laboratory-built ELS evaluation system.

and a computer, as shown in Fig. 1. The applied tensile load and loading speed were controlled to be approximately $1000 \mu$ st of strain and $1000 \mu \mathrm{st} / \mathrm{s}$ of strain rate, respectively. For quantitative and reproducible measurement, the SAOE film was once irradiated by ultraviolet light (UV, $365 \mathrm{~nm}$, $0.7 \mathrm{~mW} / \mathrm{cm}^{2}$ ) for $1 \mathrm{~min}$ and kept under dark condition for $1 \mathrm{~min}$. The ELS intensities were estimated from the ELS images.

\subsection{Material characterizations}

The crystal structure of the synthesized powders was determined by means of X-ray diffraction (XRD, RINT2000, Rigaku Co., Japan) analysis. The $\mathrm{CuK} \alpha$ radiation $(\lambda=1.5418 \AA)$ and $2.00 \% \mathrm{~min}$ angle step were used for all measurements. Morphology of the synthesized powders was observed by a field-emission scanning electron microscope (FE-SEM, JSM-7001F, JEOL Ltd., Japan). To determine the oxidation state of Eu in the samples, X-ray absorption near edge spectroscopy (XANES) was performed at beamline (BL06) in the SAGA Light Source (SAGA-LS), Japan, which has a storage ring operating energy of $1.4 \mathrm{GeV}$. Eu $L_{3}$-edge XANES spectra of the synthesized samples and reference compounds were measured under transmission mode by using pelletized mixtures $\left(10 \mathrm{~mm} \phi \times 0.5 \mathrm{~mm}^{t}\right)$ of the synthesized SAOE and boron nitride powders (BN, Kojundo Chemical Laboratory Co., Ltd., Japan). The incident intensity $\left(I_{0}\right)$ and the transmitted intensity $(I)$ through the sample were monitored using $50 \% \mathrm{He}+50 \% \mathrm{~N}_{2}$ and $15 \% \mathrm{Ar}+85 \% \mathrm{~N}_{2}$ filled ionization chambers, respectively. The obtained XANES spectra were compared with two reference spectra $\left(\mathrm{Eu}_{2} \mathrm{O}_{3}\right.$ and $\left.\mathrm{EuS}\right)$ using Athena software. ${ }^{18,19}$

The emission and excitation spectra of photoluminescence (PL) for the synthesized SAOE powders were measured via a spectrofluorometer (FP-6600, JASCO Co., Japan). The thermoluminescence (ThL) glow curve measurements were performed by means of a special system consisting of a spectrofluorometer (FP-6500, JASCO Co., Japan) and a heating/freezing stage (Linkam Scientific Instruments Ltd., UK). After deexcitation process, which a metal holder loaded with weighed amount of SAOE powder $(200 \mathrm{mg})$ was heated up to $300^{\circ} \mathrm{C}$ to release all carriers trapped in defect inside $\mathrm{SrAl}_{2} \mathrm{O}_{4}$ matrix, the $\mathrm{SAOE}$ powder was exposed to UV light for $5 \mathrm{~min}$ and waited for $1 \mathrm{~min}$ at room temperature, and then 
the ThL glow curve was recorded when it was heated up to $300^{\circ} \mathrm{C}$ at the heating rate of $30^{\circ} \mathrm{C} / \mathrm{min}$.

\section{Results and Discussion}

Figure 2(a) shows XRD patterns of the synthesized SAOE powders after sintering at $1350{ }^{\circ} \mathrm{C}$ in $5 \% \mathrm{H}_{2} / \mathrm{Ar}$ atmosphere. To calibrate the obtained XRD patterns and confirm the diffraction peak shift, a standard reference $\mathrm{CeO}_{2}$ powder (SRM $674 \mathrm{a}$, NIST) was mixed with each of the synthesized SAOE powders. As a result, both SAOE powders were in excellent agreement with the diffraction pattern of monoclinic $\mathrm{SrAl}_{2} \mathrm{O}_{4}$ (PDF no. 01-074-0794 in ICDD), and no impurity phase was observed. Figure 2(b) depicts the enlarged XRD patterns in the 2 theta range of $26-34^{\circ}$. It can be seen that all diffraction

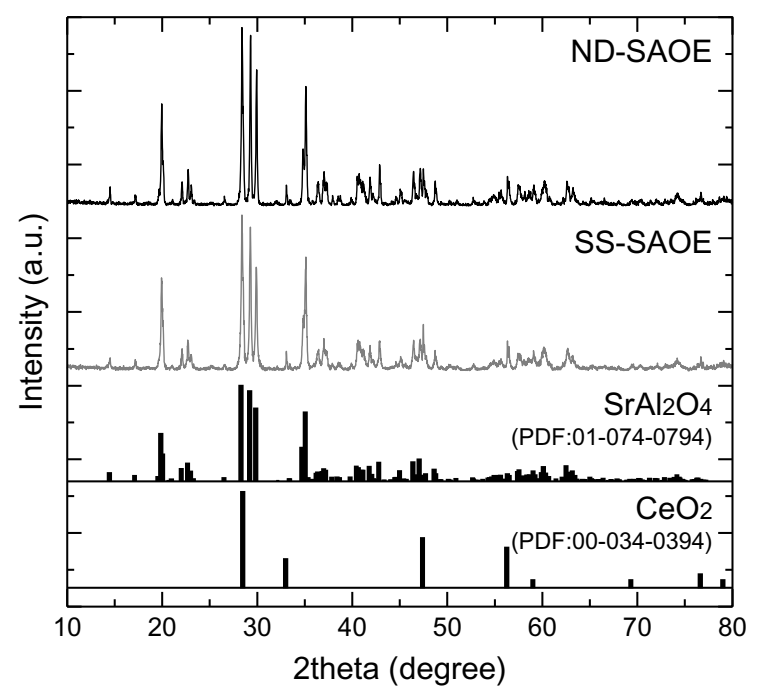

(a)

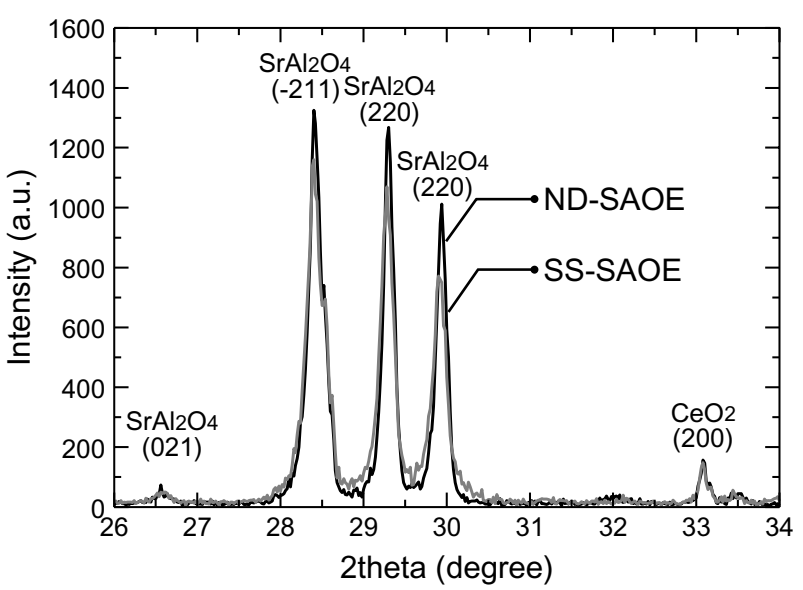

(b)

Fig. 2. (a) XRD patterns of $\mathrm{SrAl}_{2} \mathrm{O}_{4}: \mathrm{Eu}$ powders synthesized by solid-state reaction and nitrate decomposition methods as well as standard PDF data for $\mathrm{SrAl}_{2} \mathrm{O}_{4}$ (01-074-0794), and (b) enlarged $\mathrm{XRD}$ patterns of the synthesized samples.

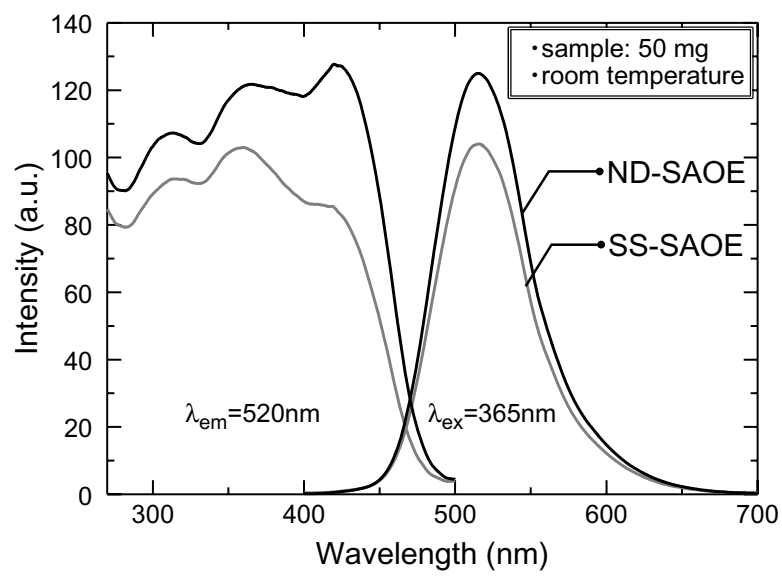

Fig. 3. Emission spectra under $365 \mathrm{~nm}$ excitation wavelength and excitation spectra under $520 \mathrm{~nm}$ emission wavelength for the synthesized SAOE powder samples.

peaks of both SAOE powders coincide completely, although the peak intensities of ND-SAOE are higher than those of SS-SAOE. This indicates that the SS-SAOE and ND-SAOE powders formed a same $\mathrm{SrAl}_{2} \mathrm{O}_{4}$ matrix, whereas the crystallinity of ND-SAOE is higher than that of SS-SAOE.

The PL emission and excitation spectra for the synthesized SAOE powders were investigated via a spectrofluorometer and compared in Fig. 3. As a result, ND-SAOE powder was found to exhibit higher excitation efficiency in the range of 400-500 $\mathrm{nm}$ compared to SS-SAOE. Such a high excitation efficiency in visible region is a practical advantage as a ML sensor. On the other hand, the peak positions of emission spectra for both SAOE powders are almost same, which is centered at approximately $520 \mathrm{~nm}$, although those PL intensities are a bit different. This suggests that PL from both synthesized SAOE powders can be attributed to the transition of electrons in $\mathrm{Eu}^{2+}$ ions from the excited state $4 f^{6} 5 d^{1}$ to the ground state of $4 f^{7}$.

To investigate the oxidation state of Eu doped in $\mathrm{SrAl}_{2} \mathrm{O}_{4}$ matrix, Eu $L_{3}$-edge XANES spectra for the synthesized SAOE powders were measured using synchrotron radiation at SAGA-LS. XANES measurement using synchrotron radiation helps in identifying an oxidation state of infinitesimal amount of dopant element. Figure 4 displays the XANES spectra of the synthesized SAOE samples and the reference compounds. The reference samples $\left(\mathrm{EuS}\right.$ and $\mathrm{Eu}_{2} \mathrm{O}_{3}$ ) gave only a peak at around 6973 and $6981 \mathrm{eV}$, respectively. The resonance for $\mathrm{Eu}^{2+}$ is approximately $8 \mathrm{eV}$ below the $\mathrm{Eu}^{3+}$ edge, which is good agreement with previous reports. ${ }^{20,21}$ The XANES spectra of both SAOE powders have only a peak which centered at approximately $6973 \mathrm{eV}$. Thus, it can be concluded that $\mathrm{Eu}$ ion doped in $\mathrm{SrAl}_{2} \mathrm{O}_{4}$ matrix for both synthesized SAOE powders exists as a divalent ion $\left(\mathrm{Eu}^{2+}\right)$.

The ELS characteristics of the fabricated ML sensors utilizing SS-SAOE and ND-SAOE films prepared by solid-state reaction method and nitrate decomposition method, respectively, were investigated under laboratory-built ELS evaluation 


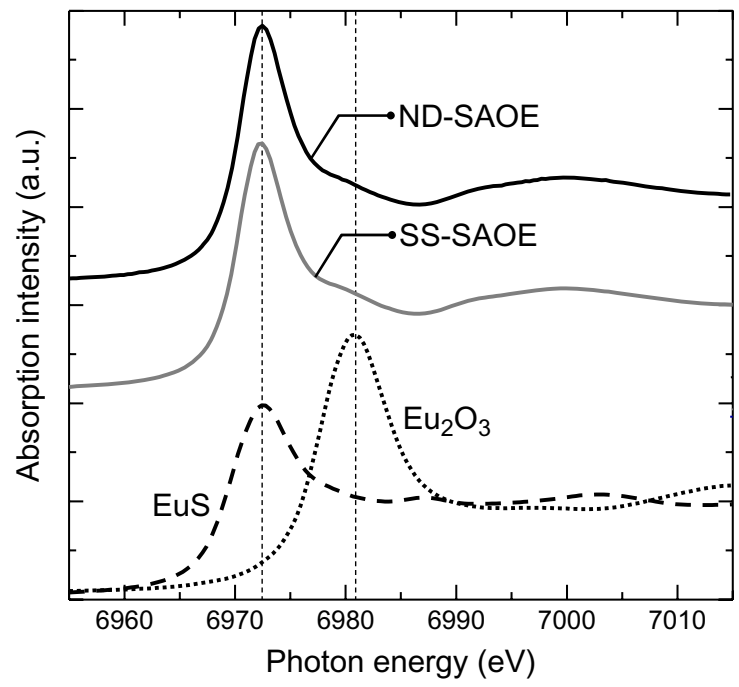

Fig. 4. Eu $L_{3}$-edge XANES spectra of the synthesized SAOE powders as well as $\mathrm{EuS}$ and $\mathrm{Eu}_{2} \mathrm{O}_{3}$ used as reference materials.

system. Figure 5 shows dependence of the ELS intensity of the ML sensors utilizing SS-SAOE and ND-SAOE films on strain obtained from the strain gage attached to the back side of SUS631. It can be seen that the ML sensor utilizing SS-SAOE film responds from approximately $300 \mu$ st of strain, and the ELS intensity increases with increase in strain in the examined range of 300-1000 $\mu$ st. Meanwhile, ND-SAOE film was found to respond to below $200 \mu$ st of strain and the ELS intensity in the range of 200-1000 $\mu$ st is larger compared to that of SSSAOE film. Such a highly sensitive response to small strain was confirmed to maintain over the examined 1000 cycles. Undoubtedly, the use of nitrate decomposition method as a synthesis of SAOE seems to be a very promising solution for improvement in the characteristic of small strain response for ML sensor.

To investigate the reasons for such an improvement in the ELS characteristic, the morphology observation and carrier

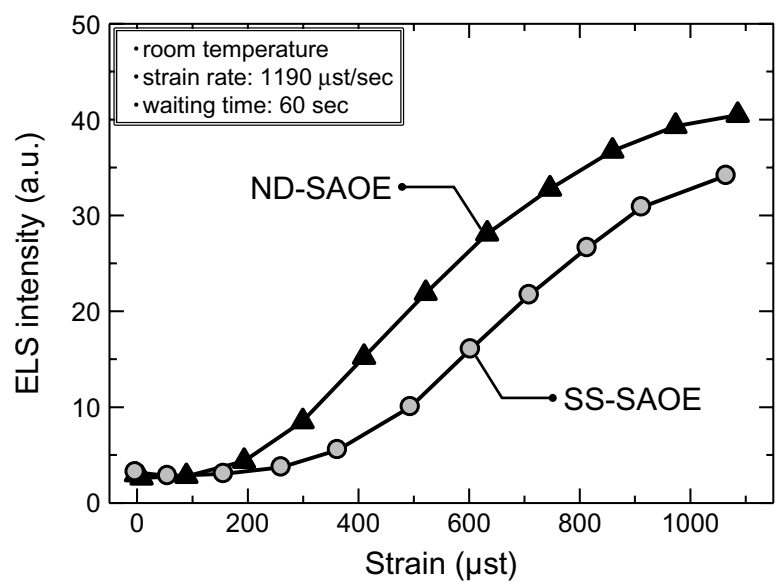

Fig. 5. Dependence of ELS intensity on strain for the ML sensors utilizing either SS-SAOE or ND-SAOE films.

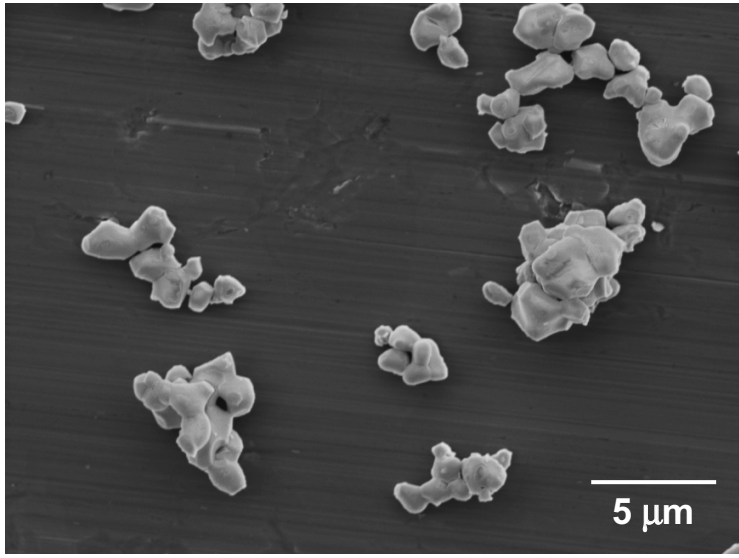

(a)

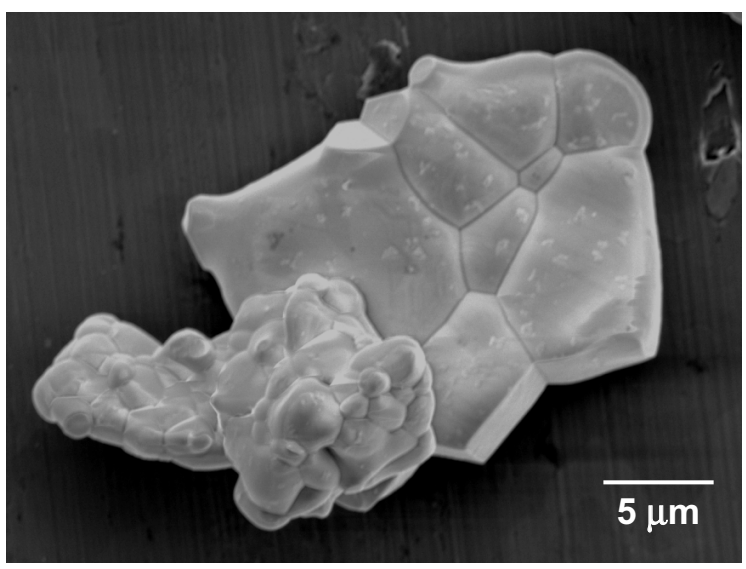

(b)

Fig. 6. Representative SEM images of (a) SS-SAOE and (b) NDSAOE powders after sintering $1350{ }^{\circ} \mathrm{C}$ in $5 \% \mathrm{H}_{2} / \mathrm{Ar}$ atmosphere.

trap level analysis for both SAOE powders were carried out by means of SEM observation and ThL measurement, respectively. Figure 6 shows the representative SEM images of the SS-SAOE and ND-SAOE powders. It is seen that the rounded SS-SAOE particles agglomerate, forming submicrometer sized SAOE particles. While, ND-SAOE particles also agglomerate, but forming much larger particles (several $10 \mu \mathrm{m}$ ). The particle shape of ND-SAOE forms plate-like and the grain boundary between primary particles is clearly seen. The size of ND-SAOE particle is larger than that of SS-SAOE particle even after sintering at same temperature. This is maybe due to a nanosized precursor in nitrate decomposition method, in other words, the much larger SAOE particle is suggested as the cause of lower melting temperature of precursor synthesized from metal nitrates. Thus, we can speculate that the improved small strain responsive ELS characteristics of ML sensor may be a result of specific shape (plate-like shape) of SAOE secondary particle formed by a nitrate decomposition method.

The mechanism of emitting light from ELS material is considered that the emission of the carriers trapped in defects. 


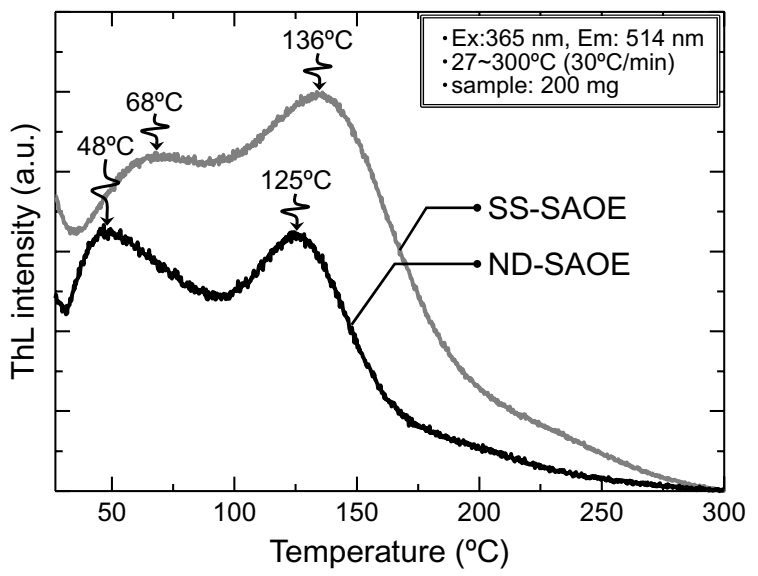

Fig. 7. Thermoluminescence glow curves for SS-SAOE and NDSAOE powders recorded at a heating rate of $30^{\circ} \mathrm{C} / \mathrm{min}$.

Considering this carrier trapping model, the trapped carrier analysis is very important for explaining the reason for improved small strain responsive characteristics of ML sensor. Thus, the trapped carrier analysis was investigated via $\mathrm{ThL}$ glow curve measurement. All of ThL glow curve measurements were carried out using a heating rate of $30^{\circ} \mathrm{C} / \mathrm{min}$ after deexcitation and excitation processes. The obtained $\mathrm{ThL}$ glow curves for both synthesized powders are shown in Fig. 7. As can be seen from Fig. 7, both SAOE powders gave two distinct peaks in $\mathrm{ThL}$ glow curves. The peaks are located at around $68^{\circ} \mathrm{C}$ and $136^{\circ} \mathrm{C}$ in the case of SS-SAOE, and around $48^{\circ} \mathrm{C}$ and $125^{\circ} \mathrm{C}$ in the case of ND-SAOE. The position of both peaks for ND-SAOE is lower than that of SSSAOE, that is to say ND-SAOE has shallower trapped level than SS-SAOE. From this result, it is indicated that the carriers trapped in defects of ND-SAOE is easy to be released by smaller thermal energy as well as smaller mechanical energy compared to SS-SAOE. Thus, the two possible mechanism of small strain response of the ML sensor using SAOE film can be proposed on the basis of the aforementioned results and considerations. This specific characteristic may be caused by a plate-like shape as well as much larger sized SAOE particle formed by a lower melting temperature of precursor, and/or shallower trapped levels, which is easy to release the trapped carrier. However, the investigation of most effective factor for small strain response should be investigated further.

\section{Conclusion}

The ELS characteristics, especially a small strain response, of the ML sensor using SAOE films synthesized by different method were examined. The ML sensor utilizing SAOE film synthesized by a nitrate decomposition method showed sensitive response to small strain (below $200 \mu$ st) compared to a conventional solid-state reaction method. Both SAOE materials show same crystal structure and the doped Eu ions in $\mathrm{SrAl}_{2} \mathrm{O}_{4}$ matrix exist as same divalent. It was revealed via
SEM observation and ThL glow curve measurement that such a sensitive response to small strain is caused by a plate-like SAOE particle and/or shallower trapped levels formed by a nitrate decomposition method.

\section{Acknowledgments}

The XANES experiments using synchrotron radiation were performed at Kyushu University Beamline (BL06) of the SAGA Light Source with the proposal of No. 2013IK021. The authors would like to thank all the members of Advanced Integrated Sensing team AIST for their valuable discussions and help in the experiment. This work was partially supported by Adaptable and Seamless Technology Transfer Program through target-driven R\&D (AS251Z02284M), Japan Science and Technology Agency as well as Grant-in-Aid for Scientific Research (A) (Grant Number: 25249100) from Japan Society for the Promotion of Science.

\section{References}

${ }^{1}$ C. N. Xu, T. Watanabe, M. Akiyama and X. G. Zheng, Artificial skin to sense mechanical stress by visible light emission, Appl. Phys. Lett. 74, 1236 (1999).

${ }^{2}$ C. N. Xu, T. Watanabe, M. Akiyama and X. G. Zheng, Direct view of stress distribution in solid by mechanoluminescence, Appl. Phys. Lett. 74, 2414 (1999).

${ }^{3}$ C. N. Xu, Encyclopedia of Smart Materials, ed. M. Schwartz, Vol. 1 (John Wiley \& Sons Inc., New York, 2002), pp. 190-201.

${ }^{4}$ J. S. Kim, Y. N. Kwon and K. S. Sohn, Dynamic visualization of crack propagation and bridging stress using the mechanoluminescence of $\mathrm{SrAl}_{2} \mathrm{O}_{4}:(\mathrm{Eu}, \mathrm{Dy}, \mathrm{Nd})$, Acta Mater. 51, 6437 (2003).

${ }^{5}$ J. S. Kim, Y. N. Kwon, N. Shin and K. S. Sohn, Mechanoluminescent $\mathrm{SrAl}_{2} \mathrm{O}_{4}$ : Eu,Dy phosphor for use in visualization of quasidynamic crack propagation, Appl. Phys. Lett. 90, 241916 (2007).

${ }^{6}$ C. Li, C. N. Xu, L. Zhang, H. Yamada and Y. Imai, Dynamic visualization of stress distribution on metal by mechanoluminescence images, J. Vis. 11(4), 329 (2008).

${ }^{7} \mathrm{D}$. Ono, C. N. Xu, C. Li and N. Bu, Visualization of internal defect of a pipe using mechanoluminescent sensor, J. Jpn. Soc. Exp. Mech. 10, 152 (2010).

${ }^{8}$ C. Li, C. N. Xu, Y. Imai and N. Bu, Real-time visualisation of the Portevin-Le Chatrlier effect with mechanoluminescent-sensing film, Strain 47, 483 (2011).

${ }^{9}$ N. Terasaki, C. N. Xu, C. Li, L. Zhang, C. Li, D. Ono, M. Tsubai, Y. Adachi, Y. Imai, N. Ueno and T. Shinokawa, Visualization of active crack on bridge in use by mechanoluminescent sensor, Proc. SPIE, Vol. 8348, (2012), pp. 83482D-1-D-6.

${ }^{10} \mathrm{~S}$. Guo, C. N. Xu, D. Ono, C. Li, Y. Sakata and S. Watanabe, Visualization of the fatigue crack for pressure vessel by mechanoluminescent sensor, 2012 IEEE Sensors Applications Symp. Proc. (2012), pp. 212-216.

${ }^{11}$ N. Ueno, C. N. Xu and S. Watanabe, Fatigue crack detection of CFRP composite pressure vessel using mechanoluminescent sensor, Proc. IEEE SENSORS 2013 (2013), pp. 1851-1854. 
${ }^{12}$ C. N. Xu, H. Yamada, X. Wang and X. G. Zheng, Strong elasticoluminescence from monoclinic-structure $\mathrm{SrAl}_{2} \mathrm{O}_{4}$, Appl. Phys. Lett. 84, 3040 (2004).

${ }^{13}$ H. Zhang, H. Yamada, N. Terasaki and C. N. Xu, Ultraviolet mechanoluminescence from $\mathrm{SrAl}_{2} \mathrm{O}_{4}: \mathrm{Ce}$ and $\mathrm{SrAl}_{2} \mathrm{O}_{4}: \mathrm{Ce}, \mathrm{Ho}$, Appl. Phys. Lett. 91, 081905 (2007).

${ }^{14}$ H. Yamada, H. Kusaba and C. N. Xu, Anisotropic lattice behavior in elasticoluminescent material $\mathrm{SrAl}_{2} \mathrm{O}_{4}: \mathrm{Eu}^{2+}$, Appl. Phys. Lett. 92, 101909 (2008).

${ }^{15}$ C. N. Xu, X. G. Zheng, M. Akiyama, K. Nonaka and T. Watanabe, Dynamic visualization of stress distribution by mechanoluminescence image, Appl. Phys. Lett. 76, 179 (2000).

${ }^{16}$ K. S. Sohn, S. Y. Seo, Y. N. Kwon and H. D. Park, Direct observation of crack tip stress field using the mechanoluminescence of $\mathrm{SrAl}_{2} \mathrm{O}_{4}$ :(Eu,Dy,Nd), J. Am. Ceram. Soc. 85, 712 (2002).
${ }^{17}$ Y. Fujio, C. N. Xu, N. Terasaki and N. Ueno, Influence of organic solvent treatment on elasticoluminescent property of europiumdoped strontium aluminates, J. Lumin. 148, 89 (2014).

${ }^{18} \mathrm{M}$. Newville, IFEFFIT: Interactive EXAFS analysis and FEFF fitting, J. Synchrotron Radiat. 8, 322 (2001).

${ }^{19}$ B. Ravel and M. Newville, ATHENA, ARTEMIS, HEPHAESTUS: Data analysis for X-ray absorption spectroscopy using IFEFFIT, J. Synchrotron Radiat. 12, 537 (2005).

${ }^{20}$ K. Korthout, K. Van den Eeckhout, J. Botterman, S. Nikitenko, D. Poelman and P. F. Smet, Luminescence and X-ray absorption measurements of persistent $\mathrm{SrAl}_{2} \mathrm{O}_{4}$ : Eu,Dy powders: Evidence for valence state changes, Phys. Rev. B 84, 085140 (2011).

${ }^{21}$ N. M. Souza-Neto, J. Zhao, E. E. Alp, G. Shen, S. V. Sinogeikin, G. Lapertot and D. Haskel, Reentrant valence transition in EuO at high pressures: Beyond the bond-valence model, Phys. Rev. Lett. 109, 026403 (2012). 